\title{
¿El alumnado que participa en violencia escolar, también participa en episodios de agresión cibernética?
}

\author{
¿Does students participating in school \\ violence also participate in cyber \\ aggression episodes?
}

\author{
¿os alunos que participam da violência \\ escolar também participam de episódios \\ de agressão cibernética?
}

\author{
Brenda Mendoza González ${ }^{1}$ \\ http://orcid.org/0000-0003-0312-5004
}

Tania Morales Reynoso \& https://orcid.org/0000-0002-8767-1098

Giovanni Martínez Gómez https://orcid.org/0000-0001-8566-0689

1 Profesor-investigador de Tiempo completo en la Universidad Autónoma del Estado de México. Miembro del Sistema Nacional de Investigadores en México (nivel 1). Línea de investigación: acoso, violencia, maltrato y abuso sexual infantil en escenarios familiares y escolares. Pos-doctora por la UNAM; Doctora en Psicología Evolutiva y de la Educación por la Universidad Complutense de Madrid (UCM), Maestra en Psicología General por la UNAM; Master en Programas de Intervención Psicológica en contextos educativos en la UCM. Fundadora de la Unidad de Atención al Maltrato y Abuso Sexual Infantil, UAMASI, de la Secretaría de Educación Pública. Publicaciones: siete libros, más de 15 capítulos de libros, y artículos en revistas científicas. Correo electrónico.: bmendozag@uaemex.mx

- Profesor-Investigador de Tiempo completo en la Facultad de Ciencias de la Conducta de la Universidad Autónoma del Estado de México. Doctora en Ciencias de la Educación por la Universidad de Santiago de Compostela. Miembro del Sistema Nacional de Investigadores, nivel 1. Líneas de investigación: violencia virtual y ciberbullying, innovación educativa, TICs y Educación. Líder del Cuerpo Académico de Psicología y Educación, forma parte del Consejo Asesor para el Observatorio de convivencia escolar, de la Secretaría de Educación Pública del Estado de México. Correo electrónico.: tmoralesr@uaemex.mx

- Licenciado en Psicología por la Universidad Autónoma del Estado de México, Maestro en psicología. Adscrito a la escuela preparatoria anexa a la normal de Tenancingo (Secretaria de Educación en el Estado de México), como coordinador en Psicología. Ponente y tallerista en Eventos Académicos. Correo electrónico.: gmg.psic@hotmail.com 
https://doi.org/10.53287/wldl7961me66a

Fecha de ingreso: 23 de febrero de 2021

Fecha de aprobación: 15 de agosto de 2021

Conflictos de intereses: Los autores declaran no tener conflictos de intereses.

\section{RESUMEN}

El acceso a la información y al conocimiento digital, es una ventaja para el desarrollo humano, sin embargo, también es una oportunidad para su uso inadecuado y desmedido. El objetivo del estudio es describir los roles de participación de violencia escolar, en función de su participación en episodios de agresión cibernética: insultos, hostigamiento, suplantación de identidad, exclusión, denigración, desvelamiento y sonsacamiento, paliza feliz, ciber-persecución y juego sucio. La investigación fue cuantitativa con estudio descriptivo y diseño transversal. Participaron 411 estudiantes de bachillerato del Estado de México. Se emplearon dos instrumentos uno para medir violencia escolar $(\alpha=.95)$ y el segundo para medir nueve tipos de agresiones cibernéticas $(\alpha=.97)$. Se hizo un análisis multivariado $k$ de medias (conglomerados) para describir a grupos de alumnos en función de la violencia escolar, identificándose tres grupos: víctimas, doble rol (víctima-acosador) y no involucrados en violencia escolar, a partir de la clasificación se hizo un contraste de medias (ANOVA de una vía) para describir los diferentes tipos de conductas ciber-agresivas exhibidas por los grupos identificados. La primera conclusión refiere que cuando se es víctima de violencia escolar (cara a cara), lo será también en ambientes virtuales: insultos cibernéticos, denigración, sonsacamiento, desvelamiento, paliza feliz, ciberpersecución, exclusión y hostigamiento. Se concluye también que quienes participan con doble rol en violencia escolar, participan como víctimas y acosadores, reproduciendo la violencia en ambientes virtuales, lo que debe tomarse en cuenta para programas de prevención y atención.

\section{PALABRAS CLAVE}

Agresión cibernética; Convivencia pacífica; Juventud; Tecnología de la comunicación; Violencia escolar.

\section{ABSTRACT}

Access to information and digital knowledge is an advantage for human development, however, it is also an opportunity for its inappropriate and excessive use. The objective of the study is to describe the roles of participation of school violence, based on their participation in episodes of cyber aggression: Flamming, Denigration, Impersonation, Outing and Trickery, Happy Slapping, Grooming, Exclusion, Cyberstalking, and Harrassment.The research was quantitative with descriptive study and cross-sectional design. 411 high school students from the State of Mexico participated. Two instruments were used, one to measure school violence $(\alpha=.95)$ and the second to measure nine types of cyber aggression $(\alpha=.97)$. A multivariate clustering o conglomerate analysis ( $k$-means) was done to describe groups of students based on school violence, identifying three groups: victims, doble role (victim-bully) and not involved in school violence, from the classification a contrast of means (one-way ANOVA) was made to describe the different types of cyber-aggressive behaviors exhibited by the identified groups. The first conclusion refers that when you are the victim of school violence (face to face), it will also be victims of school violence in virtual environments: flamming, denigration, impersonation, outing and trickery, ha- 
ppy slapping, grooming, exclusion and harassment. It is also concluded that those who participate with a doble role in school violence participate as victims and bully, reproducing violence in virtual environments, which should be taken into account for prevention and intervention program.

\section{KEY WORDS}

Cyber-aggression; Communication technology; Peaceful coexistence; School violence; Youth.

\section{RESUMO}

O acesso à informação e ao conhecimento digital é uma vantagem para o desenvolvimento humano, mas também uma oportunidade para seu uso inadequado e excessivo. O objetivo do estudo é descrever os papéis da participação na violência escolar, com base na sua participação em episódios de agressão cibernética: insultos, assédio, roubo de identidade, exclusão, difamação, divulgação e persuasão, espancamento, ciber-perseguição e groming. A investigação foi quantitativa com estudo descritivo e design transversal. Participaram 411 estudantes do ensino médio do Estado do México. Foram utilizados dois instrumentos, um para medir a violência escolar $(\alpha=, 95)$ e o segundo para medir nove tipos de ciberataques $(\alpha=, 97)$. Foi feita uma análise multivariada de meias (conglomerados) para descrever grupos de alunos baseados na violência escolar, identificando três grupos: vítimas, duplo papel (perseguidor de vítimas) e não envolvidos na violência escolar, desde a classificação um contraste de meias (ANOVA unidirecional) foi feito para descrever os diferentes tipos de comportamentos ciber-agressivos exibidos pelos grupos identificados.A primeira conclusão refere que quando se é vítima de violência escolar (cara a cara), também serão vítimas de violência escolar em ambientes virtuais: insultos cibernéticos, denegrir, sonsacing, desvendar, bater feliz, ciber-análise, exclusão e assédio. Conclui-se também que aqueles que participam com um duplo papel na violência escolar participam como vítimas e assediadores, reproduzindo a violência em ambientes virtuais, que devem ser tidos em conta para programas de prevenção e cuidados.

\section{PALAVRAS-CHAVE}

Agressão cibernética; Convivência pacífica; Tecnologia de comunicação; Juventude: Violência escolar.

\section{Introducción}

Con el objetivo de desarrollar un equilibrio mundial en las áreas sociales, económicas y medio ambientales se ha establecido una agenda internacional que permita acelerar el progreso de los países con mayor atraso, por lo que se ha determinado que una de las múltiples estrategias es el desarrollo de sociedades del conocimiento inclusivas, facilitando el acceso a las Tecnologías de la Información y Comuni- cación (TIC). En este punto de la agenda 2030, se hace hincapié en la importancia de promover ambientes educativos en el ciberespacio que se caractericen por ser saludables y libres de violencia, favoreciendo ambientes inclusivos y tolerantes propicios para el aprendizaje y la convivencia (Organización de las Naciones Unidas para la Educación, la Ciencia y la Cultura [UNESCO], 2015). 
En la actualidad se ha incrementado el uso y acceso a las TIC, por lo que las oportunidades de acceso a las tecnologías de la comunicación e información, así como acceso a internet, ha alcanzado grandes niveles de conectividad, por lo que la denominada brecha digital ha tenido que mover sus indicadores clasificándoles en tres niveles, el primer nivel señala la conexión a internet, el segundo nivel refiere el uso de este así como las competencias para emplearlo, recientemente se ha denominado al tercer nivel como los resultados de su uso, es decir, los beneficios que se obtienen por usar internet (Van Deursen y Van Dijik, 2019).

Por lo anterior, en Países Latinoamericanos la brecha digital aún no ha superado el primer nivel, ya que una tercera parte de los hogares $(33 \%)$ aún no cuentan con el servicio y el $46 \%$ de la niñez no tienen acceso a internet (Comisión Económica para América Latina y el Caribe [CEPAL] 2020). En este sentido el esfuerzo por disminuir la brecha digital ha sido considerable dentro de las instituciones educativas, sobre todo con respecto a la infraestructura necesaria para garantizar el libre acceso a la información y el conocimiento de los alumnos dentro del recinto escolar, por esta razón, son los jóvenes estudiantes los que tienen mayores oportunidades de acceso a la red. Para el caso específico de México, son un grupo poblacional cibernauta por excelencia, siendo los estudiantes del nivel superior los que registran un mayor acceso (95\%) seguidos de los estudiantes de nivel medio superior (88.5\%), (Encuesta Nacional sobre Disponibilidad y Uso de Tecnologías de la Información en los Hogares, [ENDUTIH], 2018).

Los datos con respecto al segundo nivel de la brecha digital son desalentadores, ya que aunque el conocimiento digital es una ventaja para la formación académica y el desarrollo humano personal, también presenta una oportunidad para su uso inadecuado y desmedido, y poco se ha hecho para garantizar el uso responsable de este recurso, los datos señalan que el principal fin es el del entretenimiento y comunicación (91\% y $90 \%$ respectivamente) (ENDUTIH, 2018), demostrándose recientemente que los jóvenes no son ciudadanos digitales, es decir, no tienen habilidades básicas para comprender y evaluar los contenidos e información mediática, no son críticos con respecto a problemas sociales y éticos relacionados con la tecnología, carecen de habilidades de alfabetización mediática, para comunicar, colaborar, publicar y comentar haciendo uso de la tecnología, siendo responsables éticamente para la ciber-convivencia (Mendoza, et al., 2019).

Esto también ha propiciado que no sea posible alcanzar el objetivo de la UNESCO sobre la paz y la convivencia armónica, ya que se ha identificado que el $17 \%$ de los usuarios de internet, vivió alguna agresión cibernética, siendo los adolescentes y jóvenes el grupo más vulnerable, ya que uno de cada cinco usuarios, afirma haber recibido mediante este medio, mensajes ofensivos, discriminación y han recibido insinuaciones sexuales o suplantación de su identidad (Módulo sobre Ciberacoso [MOCIBA], 2017).

\section{Marco Teórico}

En la actualidad, la investigación de violencia escolar comienza a integrar también la modalidad cibernética, lo que resulta ser un fenómeno complejo, cuya dificultad en su estudio se puede resumir en tres aspectos primordiales:

En primer lugar, existen confusiones respecto a lo que se entiende por ciberagresión y ciberacoso (o ciberbullying, en inglés), algunos investigadores consideran la ciberagresión como sinónimo del ciberacoso (Prymak y Sosnowski, 2017), entendiéndolo como el uso violento y re- 
iterado de la tecnología por parte de los estudiantes, con la intención de causar daño, y cuyo desequilibrio de poder entre las partes implicadas, hace imposible una defensa efectiva. En este caso, cualquier comportamiento que se presente se considera ciberacoso, mientras cumpla con estas características.

Pero existen otros investigadores que indican que la ciber-agresión y el ciberacoso se pueden diferenciar con precisión, al primero se le define como comportamiento negativo que se desarrolla en ambientes virtuales, independientemente de la repetición o desequilibro de poder entre los involucrados describiendo una amplia gama de comportamientos agresivos (Corcoran, et al., 2015; Smith, 2012), no importa sí el comportamiento es reiterado o solamente se manifiesta una vez, ya que en un ambiente virtual, el daño es exponencial debido a la propagación del contenido gracias a la rapidez con la que viaja la información en medios virtuales (Berkley et al., 2012; Corcoran et al., 2015; Grigg, 2010). Además, tampoco es indispensable que se produzca entre estudiantes del mismo colegio o grado escolar, sino que la versatilidad del fenómeno permite que quien sea pueda acosar y además en el momento y tiempo que sea, por lo que el término ciber-agresión resulta más amplio que el de ciberacoso.

En segundo lugar, se tiene la problemática de sus manifestaciones que son más complejas y difíciles de identificar, la mayoría de los autores indican que los comportamientos ciberagresivos son nueve, y van de menor a mayor intensidad (Álvarez et al., 2017; Bauman, 2014; Grigg, 2010; Kowalski et al., 2014; Novo et al., 2014): Insultos cibernéticos, ocurre cuando se exhibe comportamiento agresivo en ambientes virtuales, la diferencia con el insulto tradicional es que este va en escalada ya que la mayoría de los usuarios los reproduce.
Hostigamiento, es el envío reiterado de mensajes (no violentos) hacia una persona, la frecuencia y la cantidad, es lo que afecta emocional y significativamente a la víctima.

Ciberpersecución, consiste en enviar mensajes amenazantes y recurrentes a la persona con la intención de lastimarla psicológicamente, la diferencia con el hostigamiento es que en este caso la información es muy violenta e intimidante para la quien la recibe.

Suplantación, se presenta al robar la identidad virtual de una persona, haciéndose pasar por ella creando un perfil falso (con foto y nombre de la víctima), con el objetivo de afectar la integridad moral de la persona a quien eligen como víctima.

Exclusión, negar el acceso a alguien en un grupo virtual, debido al aspecto físico, ideología, género u orientación sexual, provocando daño emocional a la víctima.

Denigración, se caracteriza por evidenciar el comportamiento de alguna persona, con el objetivo de burlarse de forma exponencial a través de medios virtuales.

Desvelamiento y sonsacamiento, el agresor manipula a la víctima (quien se encuentra alienada al agresor), para que la propia víctima realice actos que son denigrantes.

Paliza Feliz, involucra varios tipos de violencia (denigración, hostigamiento, daño físico y psicológico), los agresores graban cualquier forma de agresión hacia la víctima, actos que comparten en ambientes virtuales.

Juego Sucio, ocurre cuando una persona se graba a si misma o envía sus propias imágenes o fotos con connotación sexual, que luego son distribuidas sin su consentimiento. Este comportamiento ocurre entre pares, y por lo general se presenta entre 
parejas que rompen y por venganza, el material se distribuye entre conocidos.

En este último punto, es necesario aclarar que juego sucio no es lo mismo que sexting, al sexting no se le considera en la clasificación de las ciberagresiones, pero si está tipificado como un delito cibernético, por ser una manifestación de violencia grave que dirige un adulto hacia un menor de edad.

Finalmente, en tercer lugar, tenemos el problema de la temporalidad y el espacio de la agresión. La agresión que se presenta en la vida real suele tener un tiempo y un espacio específico, es decir, en el bullying se presenta dentro de la escuela o en el trayecto hacia su casa. Sin embargo, en la ciber-agresión ésta puede darse en cualquier momento y lugar, sin que exista un lugar físico de protección para la víctima como el hogar. Además, las agresiones pueden volver a surgir ya que se retoman nuevamente después de varios meses o incluso años ya que toda la información que se sube a la red puede ser guardada y conservada para seguir acosando, por lo que los tipos de ciber-agresión son mucho más complejas que las que se presentan en el acoso tradicional, ya que son planeadas, con daño exponencial y permanente. El análisis de las características del perfil de las personas que exhiben agresión cibernética ha permitido identificar que también son violentas e impulsivas en sus relaciones cara a cara (Alvarez et al., 2018; Chatzakou, et al., 2019; Garaigordobil, 2017), se caracterizan por mostrarse constantemente tensos, ansiosos y sin control de emociones especialmente enojo (De Pasquale, 2021) que tienen gran actividad en ambientes virtuales, opinando generalmente con expresiones negativas, agresivos y de odio, para atacar especialmente temas socialmente sensibles, como feminismo, religión, política (Chatzakou, et al., 2019), pasan muchas horas diarias conectados a internet, haciendo uso de redes sociales (Festl et al., 2015; Parti et al., 2018) y el tipo de violencia más usada es la asociado al racismo, sexismo, y a la orientación sexual de las personas (Gutiérrez, et al., 2019), además son cercanas a la víctima, compañeros, amigos cercanos o parejas sentimentales (Mishna, et. al., 2018).

Actualmente, en varios países, las conductas ciberagresivas, ya tienen consecuencias legales para quien lo ejecuta, por lo que se han desarrollado políticas públicas para que existan organismos especializados (policía cibernética), que reciben y atienden las denuncias relacionadas a las agresiones cibernéticas (Comisión Nacional de Seguridad, 2019), sin embargo, la situación en contextos escolares es diferente, ya que muchas veces estas conductas no son sancionadas debido a la inexistencia de protocolos de actuación frente a estas situaciones, destacan la Ley para atender el acoso escolar en el Estado de México (2018), la guía contra el bullying de la Comisión Nacional de los Derechos Humanos (2018), o el Protocolo para actuación en caso de abuso sexual infantil, acoso escolar y maltrato en educación básica (2018).

Pero a pesar de estos esfuerzos, es necesario realizar estudios que permitan la comprensión del fenómeno en entornos escolares, que permita obtener mayor información sobre los perfiles (víctima, agresor y observador), aportando información para la prevención e intervención efectiva en la mejorar de la convivencia escolar, por lo anterior, el presente estudio pretende responder a la pregunta de investigación ¿El alumnado que participa en violencia escolar, también participa en episodios de agresión cibernética? 


\section{Objetivos de la Investigación}

\section{General}

El objetivo general de la investigación es describir los roles de participación de violencia escolar (cara a cara) en función de su participación en conductas ciber-agresivas: insultos, hostigamiento, suplantación de identidad, exclusión, denigración, desvelamiento y sonsacamiento, paliza feliz, ciber-persecución y juego sucio.

\section{Objetivos Específicos}

1. Identificar a los participantes y organizar grupos con base en su participación en violencia escolar (cara a cara).

2. Describir cada grupo de alumnos identificados (participación en violencia escolar), en función de su participación en agresión cibernética.

\section{Método}

La investigación es cuantitativa con estudio descriptivo y diseño transversal.

\subsection{Participantes}

Participaron 411 estudiantes de nivel medio superior, inscritos en el primer (43\%), segundo $(29 \%)$ y tercer año de bachillerato $(28 \%)$ de una escuela pública en el Estado de México, de ellos 183 fueron hombres (44.5\%) y 228 mujeres $(55.5 \%)$, con rango de edad de 15 a 19 años ( $\bar{x} \bar{x}$ $=16, \sigma=.98)$.

Se obtuvieron por escrito los permisos y consentimientos informados de los padres del alumnado participante. El muestreo es no probabilístico, por conveniencia (Hernández-Ávila y Carpio, 2019), lo anterior debido a un convenio de investigación con la escuela participante.

\subsection{Instrumento}

Se emplearon dos instrumentos de medición.

El primero de ellos fue el Cuestionario de Evaluación de la violencia y acoso escolar (Mendoza, et al., 2015). Tiene índice de confiabilidad total $\alpha=.95$. , consta de 85 reactivos, que miden el rol como víctima, acosador u observador en violencia escolar y bullying. Los reactivos están construidos para valorar la opinión del alumnado, en una escala Likert de cuatro niveles: todos los días, dos a tres veces por semana, dos o tres veces al mes y nunca me ha sucedido. Esta escala permite identificar la frecuencia con la que el alumnado reciben, dirigen u observan los diferentes tipos de agresión en las interacciones con los otros pares.

Los reactivos se construyeron en forma de espejo, por lo que miden el mismo tipo de agresión para cada perfil: víctima, acosador y espectador. Los diferentes niveles de agresión que mide el instrumento son exclusión (ejemplo: rechazan, ignoran); agresión de gravedad media (ejemplo: pegan, empujan, pellizcan), y agresión de gravedad extrema (ejemplo: tocan mis partes íntimas, uso de armas).

El segundo instrumento empleado fue el Cuestionario de evaluación de agresión cibernética (Morales y Mendoza, 2019), mide la frecuencia de ocurrencia de las conductas de agresión cibernética, en jóvenes entre 15 y 23 años de edad. Se conforma por 138 reactivos, que miden las respuestas con escala Likert de cinco niveles (nunca, casi nunca, algunas veces, casi siempre y siempre), con Alfa de Cronbach de 0.97. El instrumento, se divide en tres sub-escalas, con nueve factores, que evalúan conductas ciberagresivas: Agresor (46 reactivos, $\alpha=.95$ ), víctima (46 reactivos, $\alpha=.96)$, y observador (46 reactivos, $\alpha=.95$ ). 
Insultos cibernéticos (seis reactivos), son peleas acaloradas y públicas (en ambientes virtuales).

Denigración (seis reactivos), distribución de información falsa, chismes o señalamientos despectivos, a través de TIC.

Suplantación de identidad (seis reactivos), se accede al perfil de la víctima, o se crea un perfil falso para hacerse pasar por la víctima, dirigiendo agresiones a otras personas.

Paliza Feliz (seis reactivos), grabar a la víctima mientras está sufriendo alguna agresión física o burlas, el video se distribuye a través de dispositivos electrónicos y entornos en línea.

Sonsacamiento y desvelamiento, (seis reactivos), el agresor engaña a la víctima para obtener y difundir información vergonzosa y comprometedora.

Exclusión cibernética (cuatro reactivos), se ignora a la víctima al no dar respuesta a sus publicaciones, es eliminado o bloqueado de redes sociales o mensajería instantánea.

Juego Sucio (seis reactivos), enviar a través de dispositivos electrónicos y entornos en línea, contenido erótico o sexual que la propia víctima hace (a solicitud del agresor), ocurre entre pares.

Ciberpersecución (tres reactivos), son conductas violentas, intimidades y persistentes con en ambientes virtuales.

Hostigamiento cibernético (tres reactivos), enviar mensajes persistentemente para hostigar a la víctima (generalmente no violentos).

\subsection{Procedimiento}

Se presentó el proyecto a las autoridades escolares, una vez que aceptaron partici- par, se establecieron con las autoridades escolares los horarios para la aplicación de los instrumentos. Se solicitó a los padres de familia firmar el consentimiento informado para que sus hijos participarán en el proyecto de investigación.

La administración de los instrumentos se realizó en aulas escolares con la presencia del profesor de grupo y los aplicadores quienes fueron previamente capacitados para su administración. Los estudiantes contestaron el instrumento en una sesión con duración de una hora, su participación fue de forma voluntaria y se les notificó que los resultados se usarían con fines de investigación.

\subsection{Análisis de Resultados}

Para cumplir con el objetivo general de la investigación se desarrollaron los siguientes análisis estadísticos:

1. Se hizo un análisis multivariado a través de conglomerados por el procedimiento $K$ de medias, lo que permitió clasificar a los participantes con base en su participación en violencia escolar, usando los factores del instrumento de violencia escolar.

2.Para lograr describir cada grupo de alumnos identificados por su participación en violencia escolar (víctimas, doble rol y no involucrado) a partir de su participación en los nueve tipos de agresión cibernética (en cualquiera de sus roles víctima, observador u agresor), se hizo un contraste de medias a través del análisis de varianza (Anova de una vía) teniendo como factor el conglomerado obtenido y con la variable dependiente cada uno de los factores del instrumento de agresión virtual.

3. Tamaño del efecto. Se calculó el tamaño del efecto, para cada uno de los contrastes de las medias que fueron significativas (valor $p<.05)$. El cálculo se hizo a través 
del estadístico $d$ de Cohen. Para la interpretación del tamaño del efecto, se tomaron los siguientes valores: Efecto pequeño con valor $d=0.20$; efecto medio, con valor $\mathrm{d}=.50$ y tamaño del efecto grande con valor igual o superior a $\mathrm{d}=.80$.

\section{Resultados}

Clasificación del alumnado en función de violencia escolar (cara a cara)

El procedimiento $K$ de medias clasificó a los participantes en tres grupos, dichos grupos se conformaron conforme su participación en violencia escolar, se maximizó la homogeneidad intragrupo y la heterogeneidad entre los tres grupos identificados. El primer grupo denominado Doble Rol en violencia escolar, describe a 31 alumnos que representa el $9 \%$ del total de participantes, de ellos 18 son hombres (58\%) y 13 son mujeres (42\%). El alumnado de este grupo puntúa en los factores víctima y acosador en episodios de violencia escolar (doble rol), específicamente en comportamientos de rechazo y exclusión, así como pegar, burlarse, insultar, pellizcar, empujar, esconder, romper y robar pertenencias de otros, comportamientos que reciben (víctima de violencia escolar) y exhiben hacia sus compañeros de clase. Al segundo grupo se le nombró No Involu- crado y se conforma por la gran mayoría de los participantes, 214 alumnos que representa el $62 \%$ del total de participantes en el estudio. La gran mayoría de este grupo lo conforman mujeres (147, 69\%), 67 son hombres (31\%). Los participantes que integran este grupo se caracterizaron por puntuar "nunca" en la escala Likert, por lo que no participan como víctima y tampoco como acosador en las diversas formas de agresión: rechazo, exclusión, agresión grave y extrema, es decir, no se involucran en episodios de violencia escolar.

El tercer grupo identificado, se le llamó Víctima de violencia escolar, esta integrado por 100 alumnos (29\% del total de participantes), de ellos 57 son hombres ( $41 \%$ ) y 43 son mujeres (59\%). Los integrantes de este grupo se caracterizan por puntuar en la escala Likert "dos o tres veces por mes" en los reactivos que integran el factor de victimización, específicamente en los reactivos que miden rechazo y exclusión que reciben por parte de sus pares (víctima de rechazo).

En la figura 1, se representan gráficamente los grupos de alumnos identificados a través de los conglomerados: Doble rol (víctima-acosador), víctima y no involucrado en episodios de violencia escolar (cara a cara). 
Figura 1.

\section{Grupos de alumnos clasificados a través del análisis multivariado k de medias (conglomerado). Fuente: Elaboración Propia.}

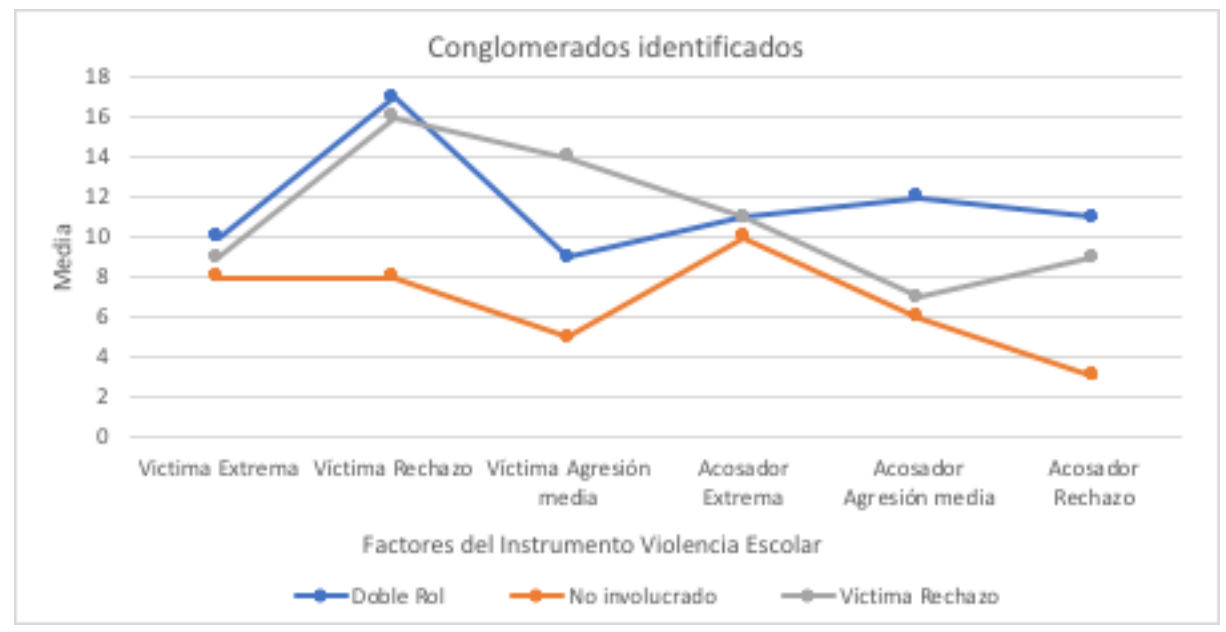

En la figura 1, se identifican los tres grupos determinados por el análisis multivariado: grupo víctima de violencia escolar, grupo doble rol (víctima-acosador) en violencia escolar y el grupo de alumnos que no se involucran en episodios violentos (cara cara) en la escuela, siendo este último el grupo con mayor representación del alumnado.

Grupos que participan en violencia escolar $y$ en conductas ciber-agresivas

A continuación, se describirá cada uno de los grupos identificados a través del análisis $k$ de medias: Doble rol (víctima-acosador), víctima y no involucrado, que representan los roles de participación en violencia escolar (cara a cara, con sus pares), la descripción permitirá conocer cual de dichos grupos participan también en episodios de agresión cibernética.

Lo anterior se logrará al examinar cuál de los grupos (doble rol, víctima o no involu- crado) presenta mayor puntaje significativo en las medias de los diferentes factores que comprenden el instrumento de agresión cibernética: insultos electrónicos, denigración, suplantación, sonsacamiento, paliza feliz, juego sucio, hostigamiento, ciber-persecución y exclusión. Para lo cual, se hizo un contraste de medias, a través de ANOVA de un factor, teniendo como factor al conglomerado (grupos identificados en violencia escolar) y como variable dependiente cada uno de los factores del instrumento de agresión cibernética.

En las tablas 1 a la 4 que a continuación se muestran, se describen los resultados de los contrastes de medias que fueron estadísticamente significativos. En cada tabla, se muestra el número de participantes que conforma cada conglomerado $(n)$, la media aritmética ( ), la desviación estándar $(\sigma)$, el valor de ANOVA $(F)$, el nivel de significancia $(p)$, los contrastes Post Hoc, y el tamaño del efecto a través del valor de la $d$ de Cohen $(d)$. 


\section{Tabla 1. \\ Contraste de medias para los factores Insultos electrónicos y Denigración}

\begin{tabular}{|c|c|c|c|c|c|c|c|c|}
\hline $\begin{array}{l}\text { Factor: } \\
\text { Instrumento } \\
\text { Agresión } \\
\text { Cibernética }\end{array}$ & $\begin{array}{l}\text { Grupos o } \\
\text { Conglomerado } \\
\text { Violencia } \\
\text { Escolar }\end{array}$ & $N$ & & $\sigma$ & $\begin{array}{c}F \\
(2 y \\
337)\end{array}$ & $p$ & Post Hoc & $d$ \\
\hline $\begin{array}{l}\text { Víctima } \\
\text { Insultos } \\
\text { Electrónicos }\end{array}$ & $\begin{array}{l}\text { Doble Rol (DR) } \\
\text { Víctima (Vi) } \\
\text { No involucrado } \\
\text { (NI) }\end{array}$ & $\begin{array}{r}31 \\
100 \\
214\end{array}$ & $\begin{array}{r}12 \\
11.38 \\
8.9\end{array}$ & $\begin{array}{l}6.64 \\
5.17 \\
4.05\end{array}$ & 1.935 & .000 & $\begin{array}{l}\mathrm{NI}<\mathrm{DR} \\
\mathrm{NI}<\mathrm{V}\end{array}$ & $\begin{array}{l}.58 \\
.54\end{array}$ \\
\hline $\begin{array}{l}\text { Agresor } \\
\text { Insultos } \\
\text { Electrónicos }\end{array}$ & $\begin{array}{l}\text { Doble Rol (DR) } \\
\text { No involucrado } \\
\text { (NI) }\end{array}$ & $\begin{array}{r}31 \\
214\end{array}$ & $\begin{array}{r}8 \\
6.68\end{array}$ & $\begin{array}{r}4.51 \\
1.8\end{array}$ & 5.688 & .000 & $\mathrm{DR}>\mathrm{NI}$ & .38 \\
\hline $\begin{array}{l}\text { Observador } \\
\text { Insultos } \\
\text { Electrónicos }\end{array}$ & $\begin{array}{l}\text { Víctima (Vi) } \\
\text { No involucrado } \\
(\mathrm{NI}) \\
\end{array}$ & $\begin{array}{l}100 \\
214\end{array}$ & $\begin{array}{l}15.72 \\
13.49\end{array}$ & $\begin{array}{l}5.62 \\
6.52\end{array}$ & 4.905 & .008 & $\mathrm{~V}>\mathrm{NI}$ & .36 \\
\hline $\begin{array}{l}\text { Víctima de } \\
\text { Denigración }\end{array}$ & $\begin{array}{l}\text { Doble Rol (DR) } \\
\text { Víctima (Vi) } \\
\text { No involucrado } \\
\text { (NI) }\end{array}$ & $\begin{array}{r}31 \\
100 \\
214\end{array}$ & $\begin{array}{r}10.58 \\
8.7 \\
7.6\end{array}$ & $\begin{array}{r}5.81 \\
3.5 \\
2.95\end{array}$ & 5.467 & .005 & $\begin{aligned} D R & >V i \\
V & >N I \\
N I & <D R\end{aligned}$ & $\begin{array}{l}.40 \\
.34 \\
.65\end{array}$ \\
\hline $\begin{array}{l}\text { Agresor de } \\
\text { Denigración }\end{array}$ & $\begin{array}{l}\text { Doble Rol (DR) } \\
\text { No involucrado } \\
\text { (NI) }\end{array}$ & $\begin{array}{r}31 \\
214\end{array}$ & $\begin{array}{l}7.51 \\
6.40\end{array}$ & $\begin{array}{l}3.32 \\
1.33\end{array}$ & 5.467 & .005 & $\mathrm{DR}>\mathrm{NI}$ & .44 \\
\hline
\end{tabular}

Fuente: elaboración propia

Los resultados de la tabla 1, señalan que el alumnado que participa en episodios de violencia escolar (cara a cara) con Doble Rol (víctima-acosador), presenta media significativamente más alta que el grupo que no se involucra (en episodios de violencia escolar), dicha media aritmética es mayor en episodios de agresiones cibernéticas como ciber-víctima de insultos electrónicos y de denigración (, respectivamente) y como ciber-agresor de insultos electrónicos y en denigración (, respectivamente).
La tabla 2 que a continuación se muestra, describe el contraste de medias, para cada grupo identificado, en la participación de episodios de violencia escolar (cara a cara, como víctima, doble rol y no involucrado), identificando cual de dichos grupos exhibe mayor participación en agresiones cibernéticas: suplantación y sonsacamiento. 
Tabla 2.

Contraste de medias para los factores: Suplantación de identidad; Sonsacamiento y desvelamiento

\begin{tabular}{|c|c|c|c|c|c|c|c|c|}
\hline $\begin{array}{l}\text { Factor: } \\
\text { Instrumento } \\
\text { Agresión } \\
\text { Cibernética }\end{array}$ & $\begin{array}{l}\text { Grupos o } \\
\text { conglomerado } \\
\text { Violencia } \\
\text { Escolar }\end{array}$ & $N$ & & $\sigma$ & $\begin{array}{c}F \\
(2 y \\
337)\end{array}$ & $P$ & $\begin{array}{l}\text { Post } \\
\text { Hoc }\end{array}$ & $d$ \\
\hline $\begin{array}{l}\text { Agresor } \\
\text { Suplantación }\end{array}$ & $\begin{array}{l}\text { Doble Rol (DR) } \\
\text { No involucrado } \\
(\mathrm{NI})\end{array}$ & $\begin{array}{r}31 \\
214\end{array}$ & $\begin{array}{l}7.03 \\
6.2\end{array}$ & $\begin{array}{l}1.94 \\
.84 \\
\end{array}$ & 5.425 & .000 & $\mathrm{DR}>\mathrm{NI}$ & .56 \\
\hline $\begin{array}{l}\text { Observador } \\
\text { Suplantación }\end{array}$ & $\begin{array}{l}\text { No involucrado } \\
\text { (NI) } \\
\text { Víctima (Vi) }\end{array}$ & $\begin{array}{l}214 \\
100\end{array}$ & $\begin{array}{l}10.6 \\
12.69\end{array}$ & $\begin{array}{l}6.01 \\
6.56\end{array}$ & 3.707 & .000 & $\mathrm{Vi}>\mathrm{NI}$ & .33 \\
\hline $\begin{array}{l}\text { Víctima } \\
\text { Sonsacamiento }\end{array}$ & $\begin{array}{l}\text { Doble Rol } \\
\text { No involucrado } \\
(\mathrm{NI}) \\
\text { Víctima }(\mathrm{Vi})\end{array}$ & $\begin{array}{r}31 \\
214 \\
100\end{array}$ & $\begin{array}{l}8.77 \\
6.5 \\
6.8\end{array}$ & $\begin{array}{l}5.81 \\
2.0 \\
1.99\end{array}$ & 10.270 & .000 & $\begin{array}{l}\mathrm{DR}>\mathrm{NI} \\
\mathrm{NI}<\mathrm{DR} \\
\mathrm{V}<\mathrm{DR}\end{array}$ & $\begin{array}{l}.52 \\
.52 \\
.45\end{array}$ \\
\hline $\begin{array}{l}\text { Agresor } \\
\text { Sonsacamiento }\end{array}$ & $\begin{array}{l}\text { Doble Rol (DR) } \\
\text { No involucrado } \\
(\mathrm{NI})\end{array}$ & $\begin{array}{r}31 \\
214\end{array}$ & $\begin{array}{l}7.1 \\
6.16\end{array}$ & $\begin{array}{l}3.18 \\
1.10\end{array}$ & 5.062 & .000 & $\mathrm{DR}>\mathrm{NI}$ & .40 \\
\hline $\begin{array}{l}\text { Observador de } \\
\text { Sonsacamiento }\end{array}$ & $\begin{array}{l}\text { Doble Rol(DR) } \\
\text { No involucrado } \\
(\mathrm{NI}) \\
\text { Víctima }(\mathrm{Vi})\end{array}$ & $\begin{array}{r}31 \\
214 \\
100\end{array}$ & $\begin{array}{l}12.51 \\
9.69 \\
12.23\end{array}$ & $\begin{array}{l}6.90 \\
5.02 \\
6.62\end{array}$ & 8.310 & .000 & $\begin{array}{l}\mathrm{DR}>\mathrm{NI} \\
\mathrm{NI}<\mathrm{DR} \\
\mathrm{NI}<\mathrm{Vi}\end{array}$ & $\begin{array}{l}.47 \\
.47 \\
.43 \\
\end{array}$ \\
\hline
\end{tabular}

Fuente: elaboración propia

Los resultados de la tabla 2 , describen a través del contraste de medias que, el alumnado que participa con doble rol (en episodios de violencia escolar, cara a cara), presenta media significativamente mayor a la del grupo de alumnos denominados no involucrado en violencia escolar. Esta media es significativamente más elevada en comportamientos ciber-agresivo como ciber-agresor en suplantación de identidad; sonsacamiento y desvelamiento ( respectivamente), como ciber-víctima de sonsacamiento y desvelamiento (, así como ciber-observador en sonsacamiento y desvelamiento.
Así mismo el rol de víctima en violencia escolar (cara a cara), presenta media significativamente mayor a la del grupo de no involucrados (también en violencia escolar), particularmente en las conductas ciber-agresivas: suplantación de identidad; sonsacamiento y desvelamiento (en el rol de ciber-observador, respectivamente).

A continuación, en la tabla 3 se muestran los contrastes de medias estadísticamente significativos, para los grupos identificados en violencia escolar (cara a cara): Doble rol, víctima y no involucrado, en función de su participación en agresiones cibernéticas: paliza feliz y juego sucio. 
Tabla 3.

Contraste de medias para los factores Paliza Feliz (happy slapping) y Juego sucio

\begin{tabular}{|c|c|c|c|c|c|c|c|c|}
\hline $\begin{array}{l}\text { Factores: } \\
\text { Instrumento } \\
\text { Agresión } \\
\text { Cibernética }\end{array}$ & $\begin{array}{l}\text { Grupos o } \\
\text { Conglomera- } \\
\text { do en Violen- } \\
\text { cia Escolar }\end{array}$ & $N$ & & $\sigma$ & $\begin{array}{c}F \\
(2 y 337)\end{array}$ & $P$ & Post Hoc & $d$ \\
\hline $\begin{array}{c}\text { Víctima } \\
\text { Paliza Feliz }\end{array}$ & $\begin{array}{c}\text { Doble Rol } \\
\text { (DR) } \\
\text { No involucra- } \\
\text { do (NI) } \\
\text { Víctima (Vi) }\end{array}$ & $\begin{array}{c}31 \\
214 \\
100\end{array}$ & $\begin{array}{l}7.70 \\
6.22 \\
6.75\end{array}$ & $\begin{array}{l}3.96 \\
1.05 \\
1.85\end{array}$ & 8.310 & .000 & $\begin{array}{l}\mathrm{DR}>\mathrm{NI} \\
\mathrm{NI}<\mathrm{DR} \\
\mathrm{Vi}>\mathrm{NI}\end{array}$ & $\begin{array}{l}.51 \\
.51 \\
.35\end{array}$ \\
\hline $\begin{array}{c}\text { Agresor } \\
\text { Paliza Feliz }\end{array}$ & $\begin{array}{c}\text { Doble Rol } \\
\text { (DR) } \\
\text { No involucra- } \\
\text { do (NI) } \\
\text { Víctima (Vi) }\end{array}$ & $\begin{array}{c}31 \\
214 \\
100\end{array}$ & $\begin{array}{l}7.33 \\
6.15 \\
6.85\end{array}$ & $\begin{array}{l}3.63 \\
.83 \\
2.23\end{array}$ & 9.580 & .000 & $\begin{array}{l}\mathrm{DR}>\mathrm{NI} \\
\mathrm{NI}<\mathrm{DR} \\
\mathrm{Vi}>\mathrm{NI}\end{array}$ & $\begin{array}{l}.45 \\
.45 \\
.42\end{array}$ \\
\hline $\begin{array}{l}\text { Observador } \\
\text { Paliza Feliz }\end{array}$ & $\begin{array}{c}\text { Doble Rol } \\
\text { No involucra- } \\
\text { do (NI) } \\
\text { Víctima (Vi) }\end{array}$ & $\begin{array}{c}31 \\
214 \\
100\end{array}$ & $\begin{array}{c}13.22 \\
9.98 \\
12.14\end{array}$ & $\begin{array}{l}7.61 \\
5.48 \\
6.24\end{array}$ & 7.005 & .003 & $\begin{array}{c}\mathrm{DR}>\mathrm{NI} \\
\mathrm{NI}<\mathrm{DR} \\
\mathrm{V}<\mathrm{NI}\end{array}$ & $\begin{array}{l}.49 \\
.49 \\
.37\end{array}$ \\
\hline $\begin{array}{c}\text { Víctima } \\
\text { Juego sucio }\end{array}$ & $\begin{array}{l}\text { Doble Rol } \\
\text { (DR) } \\
\text { No involucra- } \\
\text { do (NI) }\end{array}$ & $\begin{array}{c}31 \\
214\end{array}$ & $\begin{array}{l}7.25 \\
6.22\end{array}$ & $\begin{array}{l}3.80 \\
1.28\end{array}$ & 6.130 & .002 & $\begin{array}{l}\mathrm{DR}>\mathrm{NI} \\
\mathrm{NI}<\mathrm{DR}\end{array}$ & $\begin{array}{l}.36 \\
.36\end{array}$ \\
\hline $\begin{array}{l}\text { Observador } \\
\text { Juego sucio }\end{array}$ & $\begin{array}{c}\text { No involucra- } \\
\text { do (NI) } \\
\text { Víctima (Vi) }\end{array}$ & $\begin{array}{l}214 \\
100\end{array}$ & $\begin{array}{l}10.20 \\
12.44\end{array}$ & $\begin{array}{l}6.03 \\
6.82\end{array}$ & 5.034 & .007 & $\begin{array}{l}\mathrm{NI}<\mathrm{Vi} \\
\mathrm{Vi}>\mathrm{NI}\end{array}$ & $\begin{array}{l}.35 \\
.35\end{array}$ \\
\hline
\end{tabular}

Fuente: elaboración propia

Los resultados de la tabla 3 , señalan que existen diferencias estadísticamente significativas entre los grupos de alumnos que participan en violencia escolar (cara a cara), específicamente para el grupo de alumnos que participan con Doble rol (víctima-acosador), quienes presentan media significativamente mayor que el grupo de alumnos que no se involucran en episodios de violencia escolar, dicha media es mayor en conductas ciber-agresivas como la ciber-victimización en paliza feliz y juego sucio ( respectivamente).

La tabla 3, también señala que el grupo de alumnos con doble rol (víctima-acosador) tiene media significativamente mayor que el grupo de alumnos que no se involucran en violencia escolar, como ciber-agresor en paliza feliz y ciber-observador también en paliza feliz (.

Finalmente, las víctimas de violencia escolar, presentan media significativamente mayor que los que no se involucran en violencia escolar, específicamente como ciber-observadores en juego sucio.

En la tabla 4 que a continuación se presenta, se muestran los resultados estadísticamente significativos para el contraste de medias para los grupos que participan en violencia escolar (cara a cara): Doble rol, víctima y no involucrados, en función de su participación en episodios de agresión cibernética (exclusión, ciber-persecución y hostigamiento cibernético). 
Tabla 4.

Contraste de medias para los factores Exclusión, Ciber-persecución y Hostigamiento cibernético

\begin{tabular}{|c|c|c|c|c|c|c|c|c|}
\hline $\begin{array}{l}\text { Factores: } \\
\text { Instrumento } \\
\text { agresión } \\
\text { cibernética }\end{array}$ & $\begin{array}{l}\text { Grupos o } \\
\text { conglomera- } \\
\text { dos } \\
\text { Violencia } \\
\text { escolar }\end{array}$ & $n$ & & $\sigma$ & $\begin{array}{c}F \\
(2 y \\
337)\end{array}$ & $p$ & Post Hoc & $d$ \\
\hline $\begin{array}{l}\text { Victima } \\
\text { Exclusión }\end{array}$ & $\begin{array}{l}\text { Doble Rol } \\
\text { (DR) } \\
\text { No involucra- } \\
\text { do (NI) } \\
\text { Víctima (Vi) }\end{array}$ & $\begin{array}{l}31 \\
214 \\
100\end{array}$ & $\begin{array}{l}8.74 \\
6.31 \\
7.70\end{array}$ & $\begin{array}{l}3.79 \\
2.54 \\
2.81\end{array}$ & 15.860 & .000 & $\begin{array}{l}\mathrm{DR}>\mathrm{NI} \\
\mathrm{NI}<\mathrm{DR} \\
\mathrm{Vi}>\mathrm{NI}\end{array}$ & $\begin{array}{l}.75 \\
.75 \\
.52\end{array}$ \\
\hline $\begin{array}{l}\text { Observador } \\
\text { Exclusión }\end{array}$ & $\begin{array}{l}\text { Doble Rol } \\
\text { (DR) } \\
\text { No involucra- } \\
\text { do (NI) } \\
\text { Víctima (Vi) }\end{array}$ & $\begin{array}{l}31 \\
214 \\
100\end{array}$ & $\begin{array}{l}7.61 \\
5.99 \\
7.07\end{array}$ & $\begin{array}{l}3.89 \\
3.07 \\
3.14\end{array}$ & 6.232 & .000 & $\begin{array}{l}\mathrm{DR}>\mathrm{NI} \\
\mathrm{NI}<\mathrm{DR} \\
\mathrm{Vi}>\mathrm{NI}\end{array}$ & $\begin{array}{l}.46 \\
.46 \\
.35\end{array}$ \\
\hline $\begin{array}{l}\text { Victima } \\
\text { Ciber- } \\
\text { persecución }\end{array}$ & $\begin{array}{l}\text { No } \\
\text { involucrado } \\
\text { (NI) } \\
\text { Víctima (Vi) }\end{array}$ & $\begin{array}{l}214 \\
100\end{array}$ & $\begin{array}{l}4.40 \\
5.13\end{array}$ & $\begin{array}{l}1.80 \\
2.21\end{array}$ & 5.888 & .000 & $\begin{array}{l}\mathrm{Nl}<\mathrm{Vi} \\
\mathrm{Vi}>\mathrm{NI}\end{array}$ & $\begin{array}{l}.36 \\
.36\end{array}$ \\
\hline $\begin{array}{l}\text { Observador } \\
\text { Ciber- } \\
\text { persecución }\end{array}$ & $\begin{array}{l}\text { No } \\
\text { involucrado } \\
\text { (NI) } \\
\text { Víctima (Vi) }\end{array}$ & $\begin{array}{l}214 \\
100\end{array}$ & $\begin{array}{l}5.00 \\
5.82\end{array}$ & $\begin{array}{l}2.46 \\
2.90\end{array}$ & 4.474 & .000 & $\begin{array}{l}\mathrm{NI}<\mathrm{Vi} \\
\mathrm{Vi}>\mathrm{NI}\end{array}$ & $\begin{array}{l}.30 \\
.30\end{array}$ \\
\hline $\begin{array}{l}\text { Víctima } \\
\text { Hostigamiento }\end{array}$ & $\begin{array}{l}\text { No } \\
\text { involucrado } \\
\text { (NI) } \\
\text { Víctima (Vi) }\end{array}$ & $\begin{array}{l}214 \\
100\end{array}$ & $\begin{array}{r}.22 \\
5.16\end{array}$ & $\begin{array}{l}2.39 \\
3.53\end{array}$ & 3.961 & .001 & $\begin{array}{l}\mathrm{NI}<\mathrm{Vi} \\
\mathrm{Vi}>\mathrm{NI}\end{array}$ & .31 \\
\hline
\end{tabular}

Fuente: elaboración propia

Los resultados de la tabla 4 , señalan que el grupo de alumnos que participan en episodios de violencia escolar (cara a cara) con el doble rol (víctima-acosador) tienen media significativamente más alta que el grupo que no se involucra en violencia escolar, esta media es mayor en su participación en conductas ciber agresivas de exclusión cibernética (ciber-víctima, ciber-observador, respectivamente).

Los resultados en la tabla, también demuestran que el grupo de alumnos que participan en episodios de violencia escolar con el rol de víctima, tienen media significativamente mayor al del grupo no involucrado, en su participación en conductas de ciber-persecución como ciber-víctima y ciber-observador ( respectivamente), así como víctima de hostigamiento (.

\section{Conclusiones}

La principal conclusión de este trabajo de investigación se desprende el objetivo general y responde a la pregunta de investigación planteada, determinándose que cuando un alumno participa en violencia escolar (cara a cara) también participa en episodios de agresión cibernética con diferentes roles de participación y con diferentes tipos de agresión cibernética.

Destacándose que existe estabilidad de los roles, de esta manera el alumnado que participa con el perfil Doble rol (víc- 
tima-agresor) en episodios de violencia escolar en ambientes físicos, participa con el mismo rol en ambientes virtuales en conductas ciber-agresivas como: insultos electrónicos, denigración, sonsacamiento y desvelamiento, paliza feliz. Identificándose un cambio de rol del ambiente físico al virtual, ya que cambia de doble rol (ambiente físico) al rol de ciber-agresor en la conducta de suplantación de identidad; al rol de ciber-víctima en conductas como juego sucio y exclusión cibernética, y al rol de ciber-observador en conductas como paliza feliz y exclusión cibernética.

Con lo anterior, se afirma que el alumnado con este doble rol (víctima-agresor) en ambientes físicos (cara a cara) son quienes se encuentran en mayor riesgo de participar en múltiples conductas con diferentes perfiles en ambientes virtuales.

Con respecto al perfil de víctimas de violencia escolar (cara a cara), se concluye que también son víctimas en ambientes virtuales de conductas como: hostigamiento, ciber-persecución, participando también con el rol de observador en ambientes virtuales siendo espectador de conductas que sus pares dirigen a otros, como juego sucio y ciber-persecución.

Finalmente, el alumnado más protegido es el alumnado que no participa en episodios de violencia escolar (cara a cara, denominados no involucrados), ya que tampoco se involucra en episodios agresivos ocurridos en ambientes virtuales, es decir, es alumnado que se encuentra en menor riesgo de participar en conductas agresivas, ya que no son blanco de agresiones y tampoco las dirigen a otros, ni en ambientes físicos ni en ambientes virtuales.

\section{Discusión}

Los resultados del estudio parecen identificar como lo reportó Del Rey et al., (2012) que el patrón de comportamiento de vio- lencia en ambientes escolares físicos (cara a cara) siguen un continuo hasta llegar al comportamiento del ciberbullying, a través del cual se identifica que el mismo alumnado que participa con diferentes roles en violencia cara a cara también participan en episodios de ciberbullying, resultados que son similares a otras investigaciones internacionales sustentando así, lo encontrado en esta investigación (Álvarez, et al., 2018; Garaigordobil, 2017; Giménez et al., 2015; Larrañaga et al., 2018).

Los resultados derivados de esta investigación pueden ser explicados con una amplia gama de estudios científicos. La primera, se dirige a brindar una explicación del porque las víctimas de violencia escolar lo son también en ambientes virtuales, y por que quienes participan en violencia escolar (con doble rol, víctima-acosador) participan también en episodios agresivos en otros contextos, como el virtual.

La estabilidad del comportamiento a través del tiempo y los contextos es la primera explicación, ya que el comportamiento como víctima, agresor o doble rol (víctima-agresor) continua y además se fortalece a lo largo de las diferentes etapas por las que atraviesa el ser humano, permaneciendo con dicho perfil en cada uno de los contextos en los que socializa, por lo que si no recibe intervención psicológica especializada, el alumnado participará en episodios violentos en la familia, la escuela, el trabajo, y en su comunidad, involucrándose en interacciones violentas con diferentes personas como su pareja, amistades, familiares, vecinos, compañeros de trabajo, entre otros (Brendgen, et al., 2021; Mendoza, et al., 2021; Smith, et al., 2003).

En esta estabilidad del perfil de víctima, a través del tiempo y los contextos, influyen diversos factores, uno de ellos son las distorsiones cognitivas como el pensamiento ilusorio, que lleva a normalizar y justificar 
la violencia, por lo que no solicitan ayuda, tampoco buscan soluciones proactivas para detener el problema ya que asumen que no hay ningún conflicto que se deba resolver (Mendoza y Maldonado 2017; Moretti, y Herkovits, 2021).

Otro factor que influye para la que se mantenga el perfil de víctima, es la baja autoestima (Tintori et al., 2021) y la identificación con el estereotipo femenino tradicional, estereotipo que se fortalece y afirma aún más la victimización, al asumir que son personas débiles y vulnerables sin posibilidad de modificación conductual y cognitiva (Mendoza, et. al., 2020; Mendoza y Maldonado, 2017).

Con respecto a la estabilidad del comportamiento del perfil de acosador, que se caracteriza por lastimar desplegando diversos tipos de conductas agresivas en múltiples contextos (incluyendo el virtual), se ha explicado que dicha conducta perdura ya que, ha sido empleada exitosamente para socializar en los diferentes contextos en los que se desarrolla, como el familiar, escolar, grupos sociales de actividades recreativas o lúdicas, así como en ambientes virtuales. La conducta de agresión ha sido exitosa ya que les ha funcionado para solucionar conflictos, interactuar socialmente, logrando adquirir múltiples ganancias como, poder, liderazgo, popularidad, obtención de recursos materiales o monetarios, por lo que las personas que utilizan la agresión, la emplean debido a que no conocen otras estrategias y habilidades para socializar (Mendoza, et al., 2020; Prymak y Sosnowski, 2017; Santoyo y Mendoza, 2018).

Por otra parte, los resultados también señalan que el alumnado que participa como víctima de violencia escolar (cara a cara), participa en situaciones ciber-agresivas en ambientes virtuales con el rol de ciber-observador o con el rol de ciber-agresor. Este transitar de un rol de participación a otro, se puede explicar con base en múltiples factores involucrados en el desencadenamiento y participación de episodios agresivos con cualquier rol de involucramiento (víctima, doble rol, agresor o espectador) en cualquier modalidad (cara a cara o en ambientes virtuales). Por lo que uno de estos factores son los déficits conductuales y cognitivos que dificultan a las víctimas, espectadores y acosadores, su adaptación a los contextos en los que se desenvuelven, como por ejemplo, las distorsiones cognitivas asociadas a la percepción errónea de un ambiente diferente a lo que realmente es, por lo que pueden percibir a una persona como hostil o un ambiente agresivo cuando en realidad no lo es (Mendoza y Maldonado, 2017; Šincek et al., 2017).

Otro factor, es la carencia de habilidades sociales que les impiden relacionarse pacíficamente con los otros, como empatía, cooperación y servicio hacia los demás, mostrando menor autocontrol sobre sus emociones e impulsos (Mendoza y Maldonado, 2017; Garaigordobil, 2012), se muestran intolerantes, racistas y homofóbicos (Tintori, et al., 2021), se caracterizan por usar la agresión como estrategia de resolución de conflictos (Garaigordobil, 2017).

Así mismo, las carencias y dificultades en el ambiente familiar también son un factor que influye en el comportamiento violento, ejemplo de ello son las prácticas de crianza poco efectivas y agresivas que son empleadas en el seno familiar, asociadas a la reproducción de modelos sexistas, y el uso y justificación de la violencia (Garaigordobil y Machimbarrena, 2017; Mendoza, 2020), en sentido opuesto los lazos familiares caracterizados por honestidad y confianza disminuyen el riesgo de participar en agresiones en ambientes físicos o virtuales (Tintori, et al., 2021).

De esta manera, se destaca que las conductas ciber-agresivas no suelen presentarse por casualidad, ya que son un 
cúmulo de acciones y resultantes de la combinación de múltiples factores personales, escolares, familiares y sociales que dirigen a quien las vive, a un espiral de violencia del cual resultará difícil salir sin ayuda psicológica especializada.

Las implicaciones a nivel psicológico y psiquiátrico para quienes participan en episodios de violencia escolar en ambientes físicos o virtuales son considerables, ya que se ha identificado que facilitan el desarrollo de depresión e ideas suicidas (Echavarría et al., 2017; Iranzo, et al., 2019; Redondo, et al., 2017), así como comportamiento antisocial y delictivo (Garaigordobil, 2017).

La necesidad de comprender mejor el fenómeno de la ciber-agresión se hace urgente debido al impacto psicológico y social de quienes participan, por lo que la realización de estudios focalizados que permitan diseminar el involucramiento en agresiones cara a cara y virtuales, así como los diferentes roles en un ambiente y otro, identificando la naturaleza del fenómeno, permitirá sin duda alguna, tener elementos científicos suficientes que proveen de información para el desarrollo de estrategias eficaces de prevención y atención del fenómeno.

La importancia de este estudio es precisamente el hallazgo de las asociaciones entre los actores, identificando que participar en situaciones de violencia escolar cara a cara, sitúa al alumnado en riesgo inminente de participar también en episodios de agresiones cibernéticas, como lo son: juego sucio, paliza feliz, ciber-persecución, hostigamiento, exclusión, denigración, robo de identidad, entre otras.

Para futuras investigaciones se sugiere identificar con que grado de planificación los agresores piensan, programan y ejecutan los comportamientos agresivos para dañar a otros, así como la identificación de experimentar placer por lastimar y dominar a otros.

\section{Referencias bibliográficas}

Álvarez, D., Barreiro, A., and Núñez, J.C. (2017).Cyberaggression among Adolescents: Prevalence and Gender Differences. Comunicar, 50 (XXV), 89-97.

https://doi.org/10.3916/C50-2017-08

Álvarez, D., Núñez, J.C., García, T. and Barreiro, A. (2018). Individual, Family, and Community Predictors of Cyber-aggression among Adolescents. The European Journal of Psychology Applied to Legal Context,10 (2) 79-88. https://doi. org/10.5093/ejpalc2018a8

Bauman, S. (2014). Cyberbullying: What Counselors Need to Know. USA: John Wiley \& Sons.

Berkley, R., Beard, R. and Kaplan, D. (2012). Leveraging Diversity in a Virtual Context: Global Diversity and Cyber-Aggression. In Ch. Scott y M. Byrd (Eds), Handbook of Research on Workforce Diversity in a Global Society: Technologies and Concepts (504 -522). USA: IGI Global

Brendgen, M., Vitaro, F., Ouellet-Morin I., Dionne, G., and Boivin, M. (2021). Links Between Early Personal Characteristics, Longitudinal Profiles of Peer Victimization in School and Victimization in College or at Work. Research on Child and Adolescent Psychopathology, 49(7), 905-918. doi: 10.1007/s10802-021-00783-3.

Comisión Económica para América Latina y el Caribe [CEPAL] (2020). Universalizar el acceso a las tecnologías digitales para enfrentar los efectos del COVID-19. Santiago de Chile: Naciones Unidas.

https://repositorio.cepal.org/bitstream/ handle/11362/45938/4/S2000550_es.pdf 
Chatzakou, D., Leontiadis, I., Blackburn, J., De Cristofaro, E., Stringhini, G., Vakali, A. and Kourtellis, N. (2019). Detecting Cyberbullying and Cyberaggression in Social Media. Preprint. https://bit.ly/2OANQyF

Comisión Nacional de Derechos Humanos (2018). Contra el Bullying. Guía para docentes, Alumnado, familias y comunidad escolar de la Comisión Nacional de Derechos Humanos. México: Derechos Humanos. https://bit.ly/218AiOM

Comisión Nacional de Seguridad, (2019). Recomendaciones de la Policía Cibernética en ciberseguridad. México: Comisión Nacional de Seguridad. Recuperado de http://www.cns.gob.mx/portalWebApp/ wlp.c? $\quad \mathrm{c}=\mathrm{f} 7124$

Corcoran, L. and Mc Guckin, C. and Prentice, G. (2015). Cyberbullying or Cyber Aggression?: A Review of Existing Definitions of Cyber-Based Peer-to-Peer Aggression. Societies, 5. 245-255. https:// doi.org/10.3390/soc5020245

De Pasquale, C., Martinelli, V., Sciacca, F., Mazzone, M., Chiappedi, M., Dinaro, C. and Hichy, Z. (2021). The role of mood states in cyberbullying and cybervictimization behaviors in adolescents, Psychiatry Research, 300, https://doi.org/10.1016/j. psychres.2021.113908.

Del Rey, R. Elipe, P. y Ortega, R. (2012). Bullying and cyberbullying: overlapping and predictive value of the co-occurrence. Psicothema, 24 (4)608-613.

Echavarría, J.E., Montoya, L.E., Bernal, D.R., Rodríguez, D.M., (2017). Cyberbullying and suicidal behaviour: What is the connection? About a case. Revista Colombiana de Psiquiatria, 46 (4), 247-251. https://doi.org/10.1016/j.rcp.2016.08.004

Encuesta Nacional sobre Disponibilidad y Uso de Tecnologías de la Información en los Hogares (ENDUTIH, 2018). El Instituto Nacional de Estadística y Geografía (INEGI). Comunicado de Prensa Núm. 179/19. México: INEGI. Recuperado de: https://bit.ly/2JYS6Hj

Festl, R., Scharkow, M. and Quandt, T. (2015). The individual or the group: A multilevel analysis of cyberbullying in school classes. Human Communication Research, 41, 535-556. https://doi. org/10.1111/hcre.12056

Garaigordobil, M. (2012). Cooperative conflict-solving during adolescence: Relationships with cognitive-behavioural and predictor variables. Journal for the Study of Education and Development, 35 (2), 151-165. https://doi. org/10.1174/021037012800217998

Garaigordobil, M. (2017). Conducta antisocial: conexión con bullying/cyberbullying y estrategias de resolución de conflictos. Psychosocial Intervention, 26 (1), 47-54. http://dx.doi.org/10.1016/j. psi.2015.12.002 1132-0559

Garaigordobil. M. y Machimbarrena, J.M. (2017). Stress, competence, and parental educational styles in victims and aggressors of bullying and cyberbullying. Psicothema, 29 (3), 335-340. doi: 10.7334/psicothema2016.258.

Giménez, A., Maquilón, J., Arnaiz, P. (2015). Usos problemáticos y agresivos de las TIC por parte de adolescentes implicados en cyberbullying. Revista de Investigación Educativa, 33, (2), 335-351.

http://dx.doi.org/10.6018/rie.33.2.199841

Grigg, D. (2010). Cyber-Aggression: Definition and Concept of Cyberbullying. Journal of Psychologists and Counsellors in Schools, 20 (2), 143-156.

https://doi.org/10.1375/ajgc.20.2.143 
Gutiérrez, G., Vallejo, M. and Hernández, J. (2019). Classification of Cyber-Aggression Cases Applying Machine Learning. Applied Science, 9, (1828), 1-17.

https://doi.org/10.3390/app9091828

Hernández-Ávila, C.E. y Carpio, N. (2019). Introducción a los tipos de muestreo. Revista Alerta, 2 (1), 75-79. https://doi. org/10.5377/alerta.v2i1.7535

Iranzo, B., Buelga, S., Cava, M.J. and Ortega, J. (2019). Cyberbullying, Psychosocial Adjustment, and Suicidal Ideation in Adolescence. Psychosocial Intervention, 28 (2), 1-9.

https://doi.org/10.5093/pi2019a5

Kowalski, R., Giumetti, G., Schroeder, A., and Lattanner M. (2014). Bullying in the Digital Age: A Critical Review and Meta-Analysis of Cyberbullying Research Among Youth. Psychological Bulletin, 140 (4), 1073-1137.

https://doi.org/10.1037/a0035618

Larrañaga, E., Navarro, R., y Yubero, S. (2018). Factores socio-cognitivos y emocionales en la agresión del ciberacoso. Comunicar, XXVI (56), 19-28.

https://doi.org/10.3916/C56-2018-02

Ley para prevenir y atender el acoso escolar en el Estado de México (2018). Gaceta de Gobierno del Estado de México, México: Gobierno del Estado de México. Recuperado de https://bit.ly/2mvnAJM

Mendoza, B. (2020). El derecho a la educación, programas de intervención desde los principales agentes de cambio: padres y profesorado. En M.M.Pérez, MC Macías, E. Tapia (Ed.), Educación e Infancia (157174). Cd de México: Porrúa - UNAM.

Mendoza, B., Delgado, I., García, A. (2020). Perfil de alumnado No involucrado en bullying: descripción a partir de estereotipos de género, crianza, estrategias cognitivas-sociales y sobre-ingesta ali- mentaria. Revista Anales de Psicología, 36 (3), 483-491. DOI:

https://doi.org/10.6018/analesps.337011

Mendoza, B. y Maldonado, V. (2017). Acoso escolar y habilidades sociales en alumnado de educación básica. Ciencia Ergo-sum, 24(2), 109-116.

https://doi.org/10.30878/ces.v24n2a2

Mendoza, B. Morales, T., Serrano, C. y Serrano, J.M. (2019). Los jóvenes ¿son ciudadanos digitales?: estudio descriptivo en estudiantes de bachillerato. Revista de Psicología de la Universidad Autónoma del Estado de México, 8(15),86-100.

https://revistapsicologia.uaemex.mx/article/view/13417

Mendoza, B. Pérez, H. Domínguez, M. y Roman, M. (2021). Roles de participación en bullying y episodios violentos en la interacción profesorado-alumnado. Revista Electrónica de Investigación Educativa REDIE. En Prensa.

Mishna, F., Regehr, Ch., Lacombe, A., Daciuk, J., Fearing, G., Van Wert, M. (2018). Social media, cyber-aggression and student mental health on a university campus. Journal of Mental Health, 1, 222-229. https://doi.org/10.1080/09638237.2018.14 $\underline{37607}$

Módulo sobre Ciberacoso ([MOCIBA], 2017). Módulo experimental a la Encuesta Nacional sobre Disponibilidad y Uso de las Tecnologías de la Información y la Comunicación en Hogares, ENDUTIH, 2017. México: INEGI.Recuperado de: https:// www.inegi.org. $\mathrm{mx} / \mathrm{investigacion/ciberaco-}$ so/2017/default.html

Morales, T. y Mendoza, B (2019). Tánatos, Ares y Némesis. La representación social de la contemplación de la violencia virtual entre los jóvenes universitarios Caso: UAEMex. Proyecto de Investigación. Estado de México: Universidad Autónoma del Estado de México. 
Moretti, C. y Herkovits, D. (2021). Sobre vítimas, agressores e espectadores: uma meta-etnografia dos papéis no cyberbullying. Saúde Pública 37 (4), 1-18.https:// doi.org/10.1590/0102-311X00097120

Novo, F., Pereira, F., and Matos, M. (2014). Cyber-aggression among Portuguese Adolescents. International Journal of Cyber Criminology, 8 (2), 94-110. https://www.cybercrimejournal.com/novoetalijcc2014vol8issue2.pdf

Parti, K., Kiss, T. and Koplányi, G. (2018). Architecture of aggression in cyberspace: Testing cyber aggression in young adults in Hungary. International Journal of Cybersecurity Intelligence and Cybercrime, 1 (1), 56-68.

https://vc.bridgew.edu/cgi/viewcontent. cgi?article $=1002 \&$ context $=$ ijcic

Protocolo para la prevención, detección y actuación en caso de abuso sexual infantil, acoso escolar y maltrato en las escuelas de educación básica del SEIEM (2018). Gaceta de Gobierno. México: Gobierno del Estado de México. https://bit. Iy/2mXzSLp

Prymak, T. and Sosnowski, T. (2017). Cyber-aggression as an example of dysfunctional behaviour of the young generation in the globalized world. Studies in logic, grammar and rhetoric 52, (65), 181 - 192. https://doi.org/10.1515/slgr-2017-0050

Redondo, J., Luzardo-Briceño, M., García-Lizarazo, K. L. e Inglés, C. J. (2017). Impacto psicológico del ciberbullying en estudiantes universitarios: un estudio exploratorio. Revista Colombiana de Ciencias Sociales, 8(2), 458-478.

http://dx.doi.org/10.21501/22161201.2061

Santoyo, C. y Mendoza, B. (2018). Behavioral Patterns of Children Involved in Bullying Episodes. Frontiers in Psychology, https://doi.org/10.3389/fpsyg.2018.00456
Šincek, D. , Duvnjak, I., Milić,M. (2017). Psychological Outcomes Of Cyber-Violence on Victims, Perpetrators And Perpetrators/Victims. Hrvatska revija za rehabilitacijska istraživanja. 53, (2), 98-110. https://doi.org/10.31299/hrri.53.2.8

Smith, P.K. (2012). Cyberbullying and Cyber Aggression. In S. Jimerson, A. Nickerson, M. Mayer and M. Furlong (Eds). Handbook of School Violence and School Safety: International Research and Practice (93-103). U.K.: Routledge.

Smith P.K., Singer M., Hoel, H., and Cooper, C.L. (2003). Victimization in the school and the workplace: are there any links? British Journal Psychology,94(2),175-88. doi: $10.1348 / 000712603321661868$.

PMID: 12803813.

Tintori,A. Ciancimino, G., Giovanelli, G. y Cerbara, L. (2021). Bullying and CyberbuIlying among Italian Adolescents: The Influence of Psychosocial Factors on Violent Behaviours. International Journal of Environmental Research and Public Health, 18, 1558-1570. https://doi.org/10.3390/ ijerph18041558

UNESCO. (2015). Educación 2030: Declaración de Incheon y Marco de Acción para la realización del Objetivo de Desarrollo Sostenible 4. México: UNESCO. Recuperado el 08 de mayo de 2019, de: http://xurl. es/etyev

Van Deursen A. and Van Dijik, J. (2019). The first-level digital divide shifts from inequalities in physical access to inequalities in material access. New media and society, 21 (2), 354-375. https://doi.or$\mathrm{g} / 10.1177 \% 2 \mathrm{~F} 1461444818797082$ 